\title{
Heartbeat: Glycaemic control and excess risk of major coronary events in type 1 diabetes
}

doi:10.1136/heartjnl-2017-312470

Individuals with diabetes are known to be at increased risk of cardiovascular events. However, most studies to date have focused patients with type 2 diabetes. In this issue of Heart, Matuleviciene-Anängen and colleagues ${ }^{1}$ report the findings of a study that investigates the risk of myocardial infarction in patients with type 1 diabetes compared with matched controls in Sweden. The focus on type 1 diabetes is particularly valuable given that (unlike type 2 diabetes) its diagnosis commonly precedes the development of other potentially confounding or mediating risk factors for cardiovascular disease. With about 1500 cardiovascular events during 8 years of follow-up, the researchers show that patients with type 1 diabetes have a four times higher risk of non-fatal myocardial infarction or coronary death. The excess risk was diminished but did not disappear in those with good glycaemic control or when there was no renal dysfunction at baseline. In addition, women were found to be at higher risk of cardiovascular events at every age category investigated (figure 1).

In an accompanying editorial, Herrington and $\mathrm{Bragg}^{2}$ point out that the 4-times higher relative risk observed in this study may in fact be an underestimate, given that Sweden has been the 'top provider of diabetes care in Europe'. They further note that the present study takes the previously observed differences in outcomes between men and women with diabetes further and shows that baseline renal dysfunction is not a major determinant of the poorly understood sex differences. They also remind us that considering the wider evidence, it would be reasonable to recommend early treatment with renin-angiotensin system inhibitors and statins to curb the risk of cardiovascular disease in patients with type 1 diabetes.

There is little data on the incidence of valvular heart disease (VHD). Using data from Swedish hospital registries from

\footnotetext{
${ }^{1}$ George Institute, University of Oxford, Oxford, UK ${ }^{2}$ Division of Cardiology, University of Washington, Seattle, Washington, USA
}

Correspondence to Professor Catherine M Otto, Division of Cardiology, University of Washington, Seattle, Washington, USA; cmotto@uw.edu

Kazem Rahimi, ${ }^{1}$ Catherine M Otto ${ }^{2}$

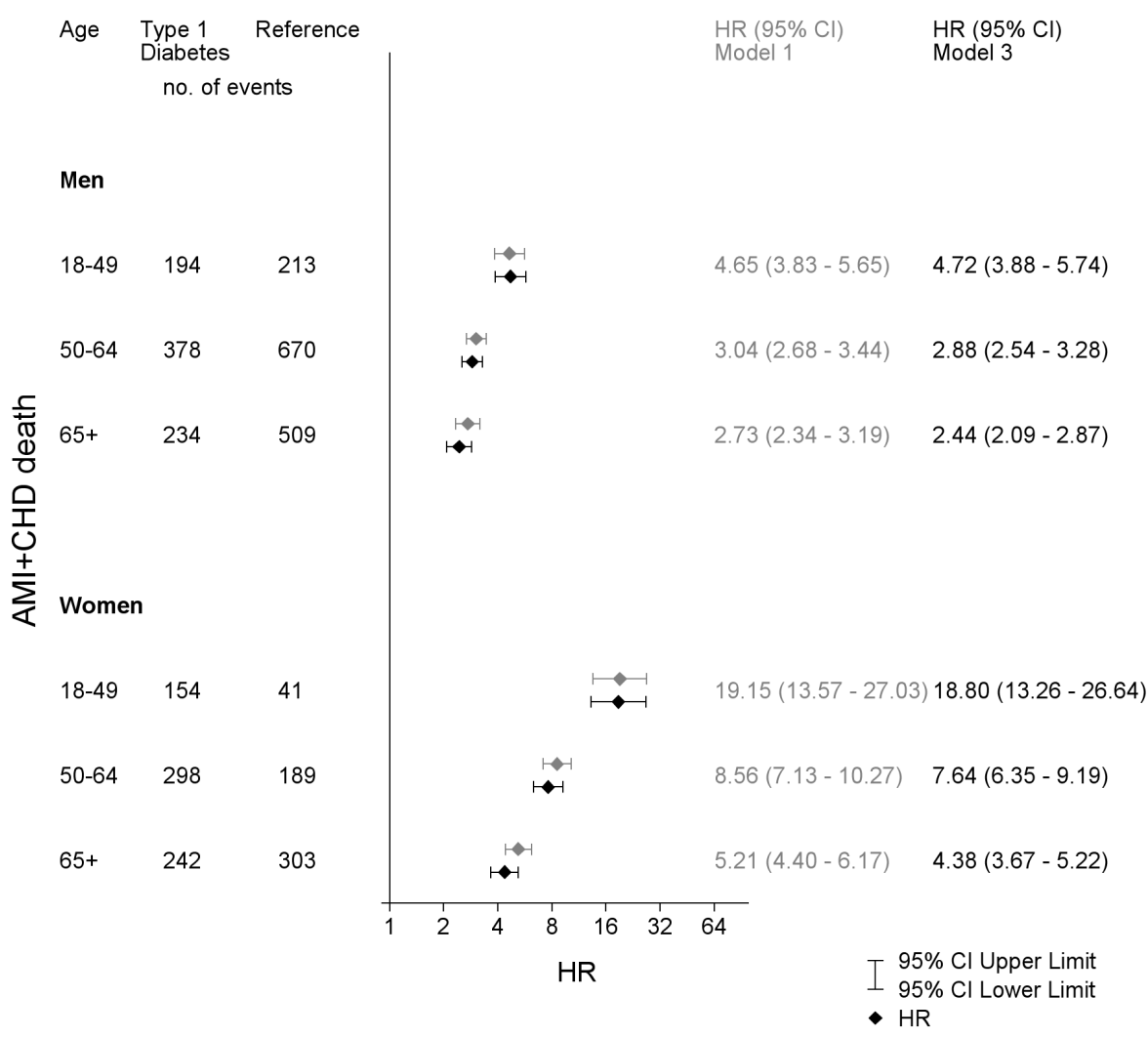

Figure 1 Adjusted HRs for acute myocardial infarction (AMI) or coronary heart disease (CHD) death and $95 \% \mathrm{Cl}$ for patients with type 1 diabetes vs the reference group examined by Cox regression. Model 1 is adjusted for time-updated age and sex, model 3 is adjusted for timeupdated age, sex, diabetes duration, born in Sweden, education level, baseline comorbidities (atrial fibrillation, CHD, heart failure, valve disease, stroke and cancer).

2003 to 2010, Andell and colleagues ${ }^{3}$ report that the incidence of VHD was 64 per 100000 person-years. Aortic stenosis was the most common type of valve disease $(47 \%)$, followed by mitral regurgitation (24\%) and aortic regurgitation (18\%). About two-thirds of all incident VHD cases were diagnosed in those aged 65 years and more (figure 2). In patients with aortic stenosis, the odds ratios compared with patients without valvular heart disease for comorbidities were 2.33 for atherosclerotic vascular disease, 5.64 for aortic aneurysm or dissection, 2.60 for heart failure, 1.60 for atrial fibrillation and 1.52 for an autoimmune disorder. In patients with aortic regurgitation the risk ratio for aortic dissection or aneurysm was 34 (CI 31.79 to 36.53 ). In patients with mitral regurgitation, odds ratios were 3.88 for atrial fibrillation, 4.93 for heart failure and 9.90 for a heritable connective tissue disorder. Rheumatic heart disease was rare but strongly associated with mitral stenosis, although $24.5 \%$ of mitral stenosis cases also had an autoimmune disease. All types of valve disease were associated with a high risk of endocarditis.

In an editorial, Clavel, Iung and

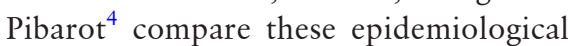
findings to other studies pointing out that incidence rates in some studies were 

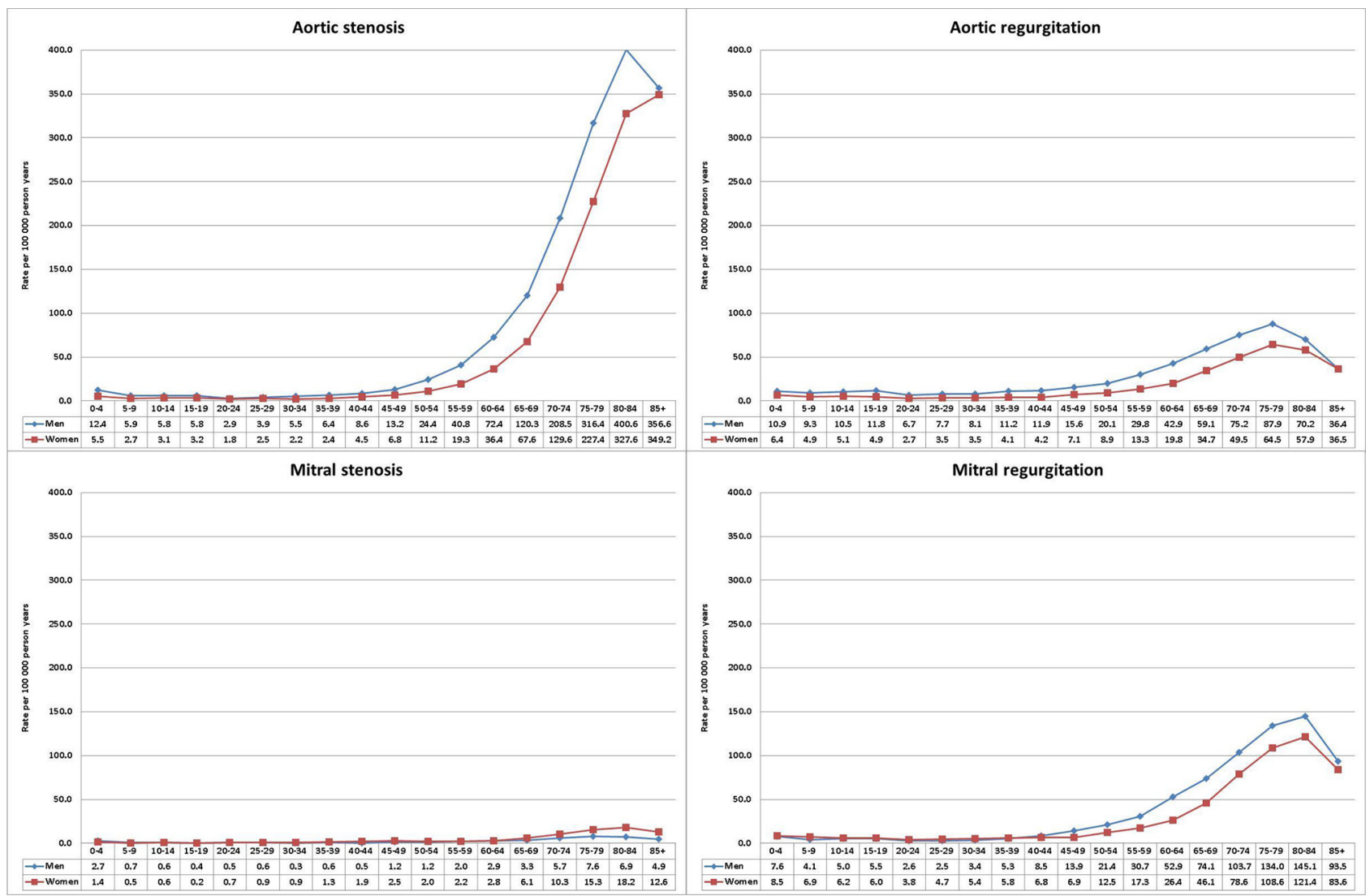

Figure 2 Incidence of left-sided valvular heart diseases in Sweden between 2003 and 2010 stratified by age and sex.

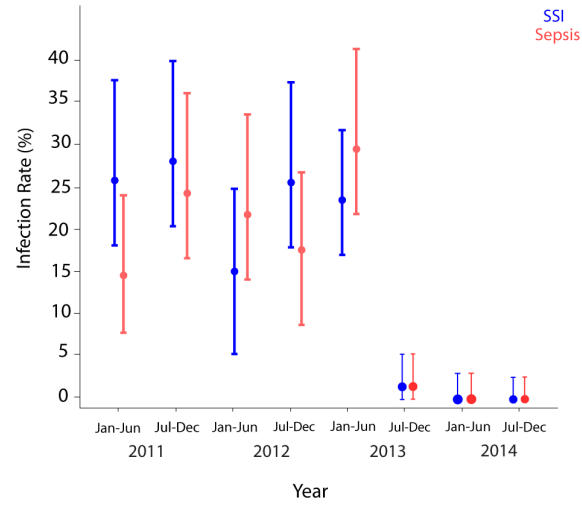

Figure 3 Interval plots representing 6 monthly infection rates. The circles represent point estimates and the ends depict $95 \%$ Cls. SSI, surgical site infection.

based on echocardiographic screening, which identifies subclinical disease, whereas the current study included only patients with a hospitalisation for valvular heart disease. They also note the sex differences in types of valve disease. Women more frequently have mitral stenosis, pulmonic stenosis and tricuspid regurgitation. Men have a higher incidence of aortic regurgitation and aortic stenosis, likely related to the higher prevalence of bicuspid valve disease in men, and have a higher incidence of mitral regurgitation compared with women. They conclude: "Given the rapid ageing of the population in developed countries, this burden and its associated impact on healthcare systems are thus expected to increase markedly in the next decades."

Diagnosis and treatment of congenital heart disease (CHD) in high-income countries has dramatically improved outcomes in these patients but these interventions are not always easily translatable worldwide. To help bridge this gap, the international Quality Improvement Collaborative (IQIC) provides guidelines aimed at reducing mortality and morbidity due to CHD surgery in low-middle income countries. In a study of post-operative CHD surgery outcomes at centres in Pakistan before and after institution of IQIC guidelines, surgical site infection and bacterial sepsis was reduced from $30 \%$ to $1 \%$ (figure 3$).^{5}$ Although the decrease in overall mortality rate from $9 \%$ to $6 \%$ was not statistically significant, there was a reduction in average duration of ventilation and length of stay for both the intensive care unit and overall hospital stay.
Yacoub and colleagues ${ }^{6}$ provide additional suggestions about strategies to improve outcomes with congenital heart disease surgery in low/middle income countries, including that "the preoperative condition of the child, both in terms of nutrition and pre-existing pulmonary, cardiovascular or systemic infection, can be one of the important predictors of outcome. This can be improved by strategies targeting the socioeconomic status of the population, early referral and intensive preoperative corrective therapy." They conclude: "improving results of congenital heart surgery in low/middle-income countries, by climbing mount Excellence, is achievable, in the foreseeable future, but requires a collaborative organised effort and a strong will by all concerned."

The Education in Heart article in this issue is on how to interpret an echocardiography report (for the non-imager). ${ }^{7}$ Practical tables and flow charts provide criteria for severity of valve disease, grading of diastolic dysfunction, and the key echocardiographic variables for each type of cardiac disease (figure 4).

Check out the Image Challenge case with Doppler data and a computed tomographic image in a patient with 


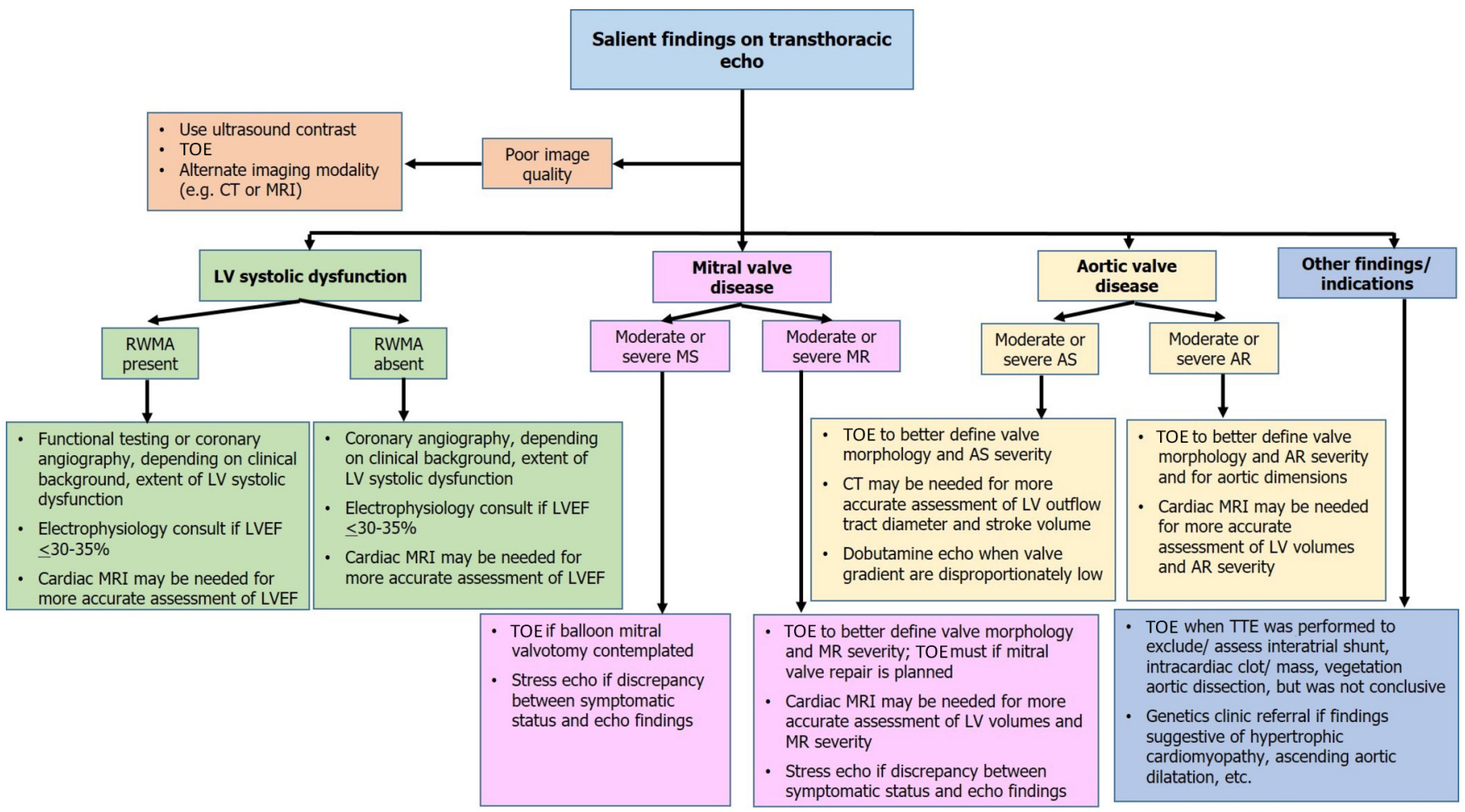

Figure 4 Approach to referral for further diagnostic evaluation based on common echocardiographic findings. AR, aortic regurgitation; AS, aortic stenosis; echo, echocardiography; LV, left ventricular; LVEF, left ventricular ejection fraction; MR, mitral regurgitation; MS, mitral stenosis; RWMA, regional wall motion abnormality; TOE, transoesophageal echocardiography; TTE, transthoracic echocardiography.

breathlessness following transcatheter aortic valve implantation. ${ }^{8}$

Competing interests None declared.

Provenance and peer review Commissioned; internally peer reviewed.

(c) Article author(s) (or their employer(s) unless otherwise stated in the text of the article) 2017. All rights reserved. No commercial use is permitted unless otherwise expressly granted.

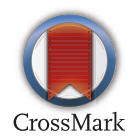

To cite Rahimi K, Otto CM. Heart 2017;103:1653-1655.
Heart 2017;103:1653-1655.

doi:10.1136/heartjnl-2017-312470

\section{REFERENCES}

1 Matuleviciene-Anängen V, Rosengren A, Svensson $\mathrm{AM}$, et al. Glycaemic control and excess risk of major coronary events in persons with type 1 diabetes. Heart 2017; 103:1687-95.

2 Herrington WG, Bragg F. Extreme ischaemic heart disease risk in people with type 1 diabetes. Heart 2017;103:1656-7.

3 Andell P, Li X, Martinsson A, et al. Epidemiology of valvular heart disease in a Swedish nationwide hospitalbased register study. Heart 2017;103:1696-703.

4 Clavel MA, lung B, Pibarot P, et al. A nationwide contemporary epidemiological portrait of valvular heart diseases. Heart 2017;103:1660-2.
5 Khan A, Abdullah A, Ahmad H, et al. Impact of International Quality Improvement Collaborative on Congenital Heart Surgery in Pakistan. Heart 2017;103:1680-6.

6 Yacoub M, Hosny H, Afifi A, et al. Improving postoperative outcome of congenital heart surgery in low/middle-income countries: climbing mount excellence. Heart 2017;103:1658-9.

7 Bansal M, Sengupta PP. How to interpret an echocardiography report (for the non-imager)? Heart 2017;103:1733-44.

8 Andrews JPM, Cruden NL, Moss AJ. Progressive breathlessness following transcatheter aortic valve replacement. Heart 2017;103:1703. 